\begin{tabular}{c} 
International Journal of Engineering \& Technology, 7 (3) (2018) 1514-1518 \\
International Journal of Engineering \& Technology \\
SPC \\
Website: $\begin{array}{c}\text { ww. sciencepubco.com/index.php/IJET } \\
\text { doi: } 10.14419 / \text { ijet.v7i3.13704 } \\
\text { Research paper }\end{array}$ \\
\hline
\end{tabular}

\title{
Application of advanced communication and control technologies for smart grid : a comprehensive review
}

\author{
S. Surender Reddy * \\ Department of Railroad and Electrical Engineering, Woosong University, Daejeon, Republic of Korea \\ *Corresponding author E-mail: surender@wsu.ac.kr
}

\begin{abstract}
This paper presents a comprehensive review of advanced control and communication technologies of smart grid (SG). Various functions/features of SG include real-time pricing, voltage/VAr control, frequency control, power quality, demand response management, distribution system restoration, and reconfiguration, distributed generation dispatch and storage. There is a strong requirement to control these functions, as well as to communicate within themselves. Suppose, if two-way communication is not possible then it cannot be considered as a function of SG. When trying to track or monitor a function, one must be able to control, command, and communicate with it. In order to control observation ability and measure of some type of numerical quantity that is obtained from a real time measuring device being moni-tored is necessary. Some of these measurable quantities are: overvoltage limits, security measures and contingency analysis. This paper addresses various challenges of control and communication techniques of SG.
\end{abstract}

Keywords: Communication; Control; Distributed Generation; Real Time Measurements; Renewable Energy; Smart Grid; Smart Meters.

\section{Introduction}

Renewable power generation is an important part of conventional as well as smart grid (SG), and it is getting more attention every day as it is economical option, pollution free and easily available in infinite amount. The SG is the next generation electrical grid, which will enable the smart integration of conventional, renewable and distributed power generation, energy storage, transmission and distribution, and demand-side management. Especially, with the advent of efficient storage options, reliability of these sources has improved. Therefore, nowadays, the renewable energy is playing a key role in generation. The renewable energy sources (RESs) can be used efficiently as distributed generation sources, which is highly motivated in SG environment. In remote and rural areas, where energy is crucial to human development, it can provide an economical platform [1]. Global warming problems and higher prices of oil and their limited availability has motivated research for wide variety of RESs that include the wind power, solar PV power, tidal wave energy, geothermal energy, biomass power and hydro generation. With the increase in population, the demand for energy production led to raise in fossil fuel based energy generation . But carbon emissions from such generation threatened the ecological balance though global warming. This forced the countries to adopted technologies that are renewable and less or zero carbon emission strategies to provide reliable electricity to all. By using the smart grid, it is easier to access electricity to the industrialization, and it improves the quality of human life and other social and economic indicators [2-3].

For control action (i.e., overvoltage), one should consider the voltage, current flowing through the components and the power factor quantities. For security measures, one should consider the speed of information that is being sent, number of components in system, voltage, current and power factor quantities. Whereas, in contingency analysis, one should consider the voltage, current, power factor, frequency, topology information and the energy consumption. For the control of smart grid (SG), we need advanced optimization methods which include real time optimality and dynamic procedures involving: stochastic programming, adaptive dynamic programming, and heuristic programming. The quantification of real time quantities follows with communications and state estimation [4]. Reference [5] discusses the rise of SG concept from the computing and communications perspective, explores the challenges related to the energy management and the communication control. The challenges related to the security of SG and the possible solutions are discussed in [6]. A review of integral components of SG and its communication infrastructures enabling the six SG applications is presented in Reference [7]. Reference [8] reviews the issues at stake and the research challenges that characterize the SGs from a security and privacy point of view are discussed and presented in [8]. The cyber security evaluation of active distribution grids characterized by a high level penetration of RESs is presented in [9]. Reference [10] provides an integrated and holistic understanding of technology-stakeholder-policy interactions of SG technologies, and also presents the state-of-the-art policy experiences and research developments. Reference [11] presents the smart metering and monitoring approach to reduce energy consumption as well as the cost. An overview of data management for SGs and the added value of Big Data technologies is presented in [12]. Reference [13] presents the effects of the development of SG and nanotechnology technologies on reducing the carbon emissions. For the increased use of communications and information technology, sufficient access to communications facilities is very important for the SG, and it has been presented in Reference [14]. Reference [15] presents the communication security aspects of SG metering and control system 
from the point of view of cryptographic techniques to enhance cyber security of the emerging SG.

From the above literature survey, it can be observed that a well functional control and communication of a typical two-way communication of the SG is necessary for better demand response, load control, etc. Load control or load adjustments made by the utility via two way communication of the SG must be non-disruptive in the sense that consumers are unaware of the control actions. In achieving this feature of the SG, a need therefore arises for an appropriate control and communication in the grid that could provide an important grid reliability enhancement, and enhancing the fundamental understanding of stability characteristics of power system.

The remainder of this paper is organized as follows: Section 2 describes various communication technologies of SG. The control technologies of SG are presented in Section 3. Section 4 presents the application of control and communication technologies to the SG in real time. Finally, the conclusions are summarized in Section 5 .

\section{Communication technologies in smart grid}

For the real time measurement, it is necessary that various quantities of SG must be applied for the efficient and effective operation. There a several devices that needs to be considered in terms of real time measurement, which includes remote terminal units (RTUs) and phasor measurement units (PMUs) [16].

\subsection{Remote terminal unit (RTU)}

The interface between objects of the physical world and Supervisory Control and Data Acquisition (SCADA) is made possible by the transmission of telemetry data to the system and utilization of messages from supervisory system to control the connected objects. Sometimes, RTUs are also known as remote telemetry units. There are many companies that provide SG solutions to utilities that utilize RTUs. Further example of how the RTU is utilized when the circuit breaker control unit integrates with an Intelligent Electronic Device (IED), it detects the fault, and then instantly trips the switchgear and blocks fault current. After that, RTU communicates the event to the distribution management system control center. The Distribution Management System (DMS) is able to take the automated or operator-initiated action in order to isolate the fault section and therefore restore the power to the functioning and healthy parts of utility grid. The RTUs collects the communication from various components of DMS including the capacitor banks, substation transformers, sectionalizer switches, etc [17].

\subsection{Phasor measurement unit (PMU)}

The PMUs can be individually devices or be functions that are incorporated into other grid components, such as protective relays. PMU technology is an advanced technology when compared to the SCADA. SCADA is an X-ray of a power grid where as PMU is a MRI. The advantages of PMUs when compared with the commonly used technology i.e., SCADA are:

- Measurements are obtained at a faster rate.

- Measurements are time synchronized.

- Provides dynamic behavior of the system.

- Situational awareness conveyed to the operators.

If synchrophasors or PMUs were implemented on the power systems, then the operators would have know the problems hours ahead the possible collapse in the system [18]

\subsubsection{Challenges of PMUs implementation}

In spite of having various advantages of PMUs, there exist several disadvantages/challenges for implementing the PMUs into the system. They include [19],

- Diverse requirements from different utilities.
- High investment: initial cost of implementing PMUs deters many utilities from installation at this point in time, and a clearly defined roadmap needs to be created or a cost benefit analysis of the implementation of PMUs will assist in the necessary deployment of this technology.

- Lack of related produce, i.e., application software.

- Visualization of PMU data: It is very difficult to visualize the numerous amount of data being recorded by PMUs.

- Communication of PMU data: The communication network required for PMUs is extremely expensive.

- Communication delays.

- Low frequency oscillation monitoring.

- Bad data estimation (i.e., state estimation (SE).

- Online voltage instability prediction.

\subsection{Output modules for smart grid}

\subsubsection{Analog to digital converters (ADCs)}

An analog voltage is transformed to a binary number, consisting of a series of 1's and 0 's, and then into a digital number, base 10. This number is then able to read on a meter, monitor, or visual display. The digital number is only an approximation of the actual value of the analog voltage at a specific instant because it can only be presented discretely. The higher the sampling rate of the digital signal, the closer to the digital signal will be to the original analog signal [20-21].

\subsubsection{Digital to analog converters (DACs)}

As from analog to digital, there is also a process to go from digital back to analog or digital code back to a current or voltage signal. Now, the digital to analog converters are mainly manufactured on integrated circuits due to cost and the need for matched components. There are various DAC architectures that have different advantages and disadvantages and the suitability of a certain DAC for a specific function or application is determined by a number of measurements, which includes speed and resolution.

Various companies are using analog to digital converters in different ways for the SG solutions as they propose to utilities and other entities. The ADCs in their SG solutions, guide in different areas such as smart meters, power-grid monitoring, communications, and energy management. A key innovation that the solution supplies is multiplexed systems versus architectures that dedicate separate ADCs to each channel that is monitoring analog signals. Smart meters create an environment where the power transactions are open and secure by reducing the risk of fraud/theft, help to manage debt recovery, to increase transparency in price changes and fees, and there by improves the efficiency [22].

The ETAP software offers various solutions dealing with the SG, from distribution and energy management systems to industry issues. One solution in particular is its state and load estimation feature. One main difference between these methods of control, are the areas they cover. For example the DMS is a system that manages of distribution systems or circuits, where an EMS manages the systems within transmission networks [23].

\subsection{Bandwidth requirements for smart grid communica- tion applications}

Bandwidth is required to accomplish best SG technology in the system. The following are the required communication technologies for enhancing a resilient and sustainable SG technology:

\subsubsection{Customer premise to aggregation point}

A SG communication network should aim to appropriately transfer to and fro the grid's information from/away the customer premises to an aggregation point, such a substation, a communications tower or a utility pole-mounted device [24]. 


\subsubsection{Distributed energy resources (DERS)}

The bandwidth (BW) required for distributed energy resources (DERs) will be along the same lines as that required for Advanced metering infrastructure (AMI). This BW requirement is allocated per each DER for a good communication networks in the grid.

\subsubsection{Real-time advanced metering infrastructure (AMI)}

For better optimal communication systems, the SG advanced metering infrastructure is best with enabled real-time control function.

\subsubsection{Distribution automation (DA)}

The particular communication path for DA will probably be on a shared medium, i.e., the communication system will not be dedicated just for DA communications to enhance the SG communication systems [25].

\subsubsection{Synchro phasor communications}

The synchrophasor communications requirements vary depending on the nature of data being transmitted. This requirement should be suited depending on the region of applications of SG communication systems.

\subsubsection{Video applications}

There are many possible uses of video applications in the SG, for example traditional video surveillance to use video cameras to relay images of analog dial readings to a control room. This means that video applications in communications infrastructure will have different requirements.

Some posed research topics in smart grid communication systems that are still being addressed include the role of sensors in the SG and their challenges, to make use the hierarchical communication architectures and the concerns regarding cyber-security being used for wireless communication. Cyber-physical security is an emerging field focusing on attacks that target the cyber and physical system components of the smart grid including phasor measurement units, intelligent electronic devices, communication links, control systems and metering devices with the ultimate goal of disrupting physical components such as generators or transmission lines. Such attacks can lead to cascading failures that also affect power flow, but employ diverse threat and attacker capability models [25].

\section{Control technologies for smart grid}

The deployment of smart grid is a revolutionary and imperative around the world. It involves and deals with lot of multidisciplinary areas like energy sources, control systems, communications, computational, generation, transmission, distribution, customer, operations, markets and service provider. Smart grids are emerging in both developed and developing countries for achieving reliable and secure electricity system to all. Further, smart grid technologies will enable the emission reductions. Smart grids will eventually need standards, policy and regulatory framework for successful implementation. Following high priority areas are focused recently in smart grid technologies: Information and communication technology integration, wide-area monitoring and control, Transmission enhancement applications, distribution and management, advanced metering infrastructure, charging infrastructure and customer side systems [26].

Since the proponents for a more sustainable power systems, i.e. smart grid (SG), numerous efforts have been embarked on by several institutions, bodies, and organizations to address the overall goal of the SG. To ensure that all features of SG are rightly implemented, the SG proponents have come up with several test beds that aims to address not only the features but also architecture. This approach has been embarked on for a while now in a bid to ensure that a deplorable SG does not in any way fall short of its features and architecture when in operation in the real world of power systems.

\subsection{Distribution automation system (DAS)}

The DAS uses remotely operated monitoring and control system of switches and breakers on the distribution system in real-time. The DAS encompasses the distribution substations and supports the optimal network operation such as fault processing, loss minimization, and load balancing. It comprises the control center, automated switches, RTUs, communication network, and modem. For the DAS to be intelligent, additional functions such as monitoring and diagnosis of equipment, power quality monitoring, and interconnection of DG to the power grid are added [27].

Distribution automation (DA) is composed of the control center, automated switches, RTUs, communication network and the modem. In the intelligent DAS (IDAS), additional functions such as monitoring and diagnosis of the equipment, power quality monitoring and interconnection of DG to the power grid are added. Usually, the control center consists of the main server, backup server and historical server. In the distribution network, several kinds of automated switches and breakers are networked through the RTUs. The communication network interconnects the RTUs in the field and control center. For the field test, the main server for IDAS, monitoring devices of the thunderbolt/lightning protector, load monitoring devices of the ground transformer are included. The main server is the integrated system with SCADA and DAS. It includes several kinds of applications, monitors and controls for the substation and the distribution feeder together. Most intelligent devices used include different kinds and they are used to monitor and measure partial discharge, temperature, gas pressure, lifespan of a circuit breaker, and power quality.

\subsection{Substation automation system (SAS)}

The SAS is the automated system that utilizes substation operation with a management system under one network. Different functions such as measurement, control, monitoring, diagnosis and protection of the power facilities in the substation are standardized, integrated and interconnected. It is composed of the intelligent electronic devices (IEDs), operating systems including human machine interfaces (HMIs) gateways, network devices such as Ethernet switches and optical cables [28].

\subsection{Intelligent transmission system}

The Intelligent Transmission System is composed of on-line monitoring system for transmission facilities, crisis management system with a satellite and reactive management system in the power grid. In on-line monitoring system for transmission facilities, different sensors are installed on the transmission monitoring network and implemented in the HMI system of the main center. This HMI system consists of the terminal devices, HMI server and the communication devices which connect with each other for data communication. The terminal devices monitor wind direction/speed, insolation, and temperature.

The Gridlink, GridIQ are some under-development hardware for the SG which can be represented at any level of the power system i.e. generation, transmission or distribution. These are currently the only good so far timing hardware simulator for the SG. The eMEGAsim, GridSpice, Gridlink, GridIQ, GridSim, and Dynamic Monitoring and Decision Making System (DYMONDS) simulator are industry ideal currently under developments platforms for realtime simulation of large power systems models including the SGs. Current researches in adaptive stochastic control for SG is aimed at addressing the control features (i.e., self-healing, flexible, predictive, interactive and optimal) inherent in SG. There is a need to protect the large amounts of classified information that will be transmitted and received wirelessly through several different types of 
networks (i.e., wide area, local and personal) by using the cyber security systems. Different types of standards are required to be considered in order to protect the system.

\section{Application of control and communication technologies to smart grid}

There are many applications for the control and communication to smart grid in real time in current use, being tested, or being developed. Many of the topics already discussed earlier in this paper have mentioned various ways in which these new technologies can be applied for the SG. For example, PMUs are being developed to the point where the measurements they record in real time can be viewed by operators, enabling them to make more accurate decisions before problems occur. RTUs are considered as the data acquisition measurement infrastructure which interfaces with various device infrastructure such as substation devices, feeder devices and customer devices. The computation infrastructure include the DMS automation and its functions include the substation automation, customer outage and billing [29].

These application areas of control and communication are the fundamental or underlying technologies for supporting power system in real time monitoring and dynamic pricing. In cost-benefit analysis for any domain of power system, mutual impacts of power system domain should be taken into account based on the holistic approach to the managements of the entire power system. The commonly used equipments for limiting over-voltages include bushings, cables, capacitors, transformers, coupling capacitors, circuit breakers, voltage transformers, surge arresters, insulators, gas insulated substations, and shunt reactors. State estimation could be used as enhancements attributed to PMUs which goes a long way to determine the best location of the PMUs. Therefore, a reliable performance index metrics is necessary to measure the progress on implementing the characteristic of optimizing asset utilization and operational efficiency of the SG. The crosscutting performance metrics at transmission, distribution and consumer ends depends on the number of smart sensors, i.e., intelligent electronic devices (IEDs) deployed, number of integrated operational information technology applications, and the number of IEDs with communications that allows the IED to perform its function.

\section{Conclusions}

This paper has presented various control and communication technologies of smart grid (SG) which can provide an important grid reliability enhancement, and to enhance the fundamental understanding of stability characteristics of power system. It also presented the cost-benefit analysis of control and communication systems in SG. From this paper, it can be observed that for the effective control and communication technologies of SG, it should has the provision to expand grid infrastructure by including the RESs, it must has good communication technology for full automation of the grid, and it should has advanced control, protection and monitoring infrastructure.

\section{Acknowledgment}

This research work is based on the support of "Woosong University's Academic Research Funding - 2018".

\section{References}

[1] N.U. Pérez, I. Angulo, D.d.1. Vega, T. Arzuaga, I. Fernández, A. Arrinda (2017), Smart Grid Applications for a Practical Implementation of IP over Narrowband Power Line Communications, Energies, vol. 10, pp. 1-16. https://doi.org/10.3390/en10111782.
[2] S. Elyengui, R. Bouhouchi, T. Ezzedine (2013), The Enhancement of Communication Technologies and Networks for Smart Grid Applications, International Journal of Emerging Trends \& Technology in Computer Science, vol. 2, no. 6, pp. 107-115.

[3] F. Ye, Y. Qian, R.Q. Hu (2018), Smart Grid Communication Infrastructures: Big Data, Cloud Computing, and Security, Wiley-IEEE Press. ISBN: 978-1-119-24015-0 https://doi.org/10.1002/9781119240136.

[4] Z. Uddin, A. Ahmad, A. Qamar, M. Altaf (2018), Recent advances of the signal processing techniques in future smart grids, Human Centric Computing and Information Sciences, vol. 8, no. 2, pp. 1-15 https://doi.org/10.1186/s13673-018-0126-9.

[5] B. Fayçal, M. Michael, M. Madjid (2014), Communication Challenges and Solutions in the Smart Grid, SpringerBriefs in Computer Science, Springer-Verlag New York. https://doi.org/10.1007/978-14939-2184-3.

[6] V. Rohokale, R. Prasad (2016), Cyber Security for Smart Grid - The Backbone of Social Economy, Journal of Cyber Security, vol. 5, pp. 55-76. https://doi.org/10.13052/jcsm2245-1439.514.

[7] M. Emmanuel, R. Rayudu (2016), Communication technologies for smart grid applications, Journal of Network and Computer Applications, vol. 74, no. C, pp. 133-148 https://doi.org/10.1016/j.jnca.2016.08.012.

[8] M.R. Asghar, D. Miorandi (2013), a Holistic View of Security and Privacy Issues in Smart Grids. In: Cuellar J. (eds) Smart Grid Security, SmartGridSec 2012, Lecture Notes in Computer Science, vol. 7823, Springer, Berlin, Heidelberg, pp 58-71. https://doi.org/10.1007/978-3-642-38030-3 4.

[9] G. Dondossola, R. Terruggia (2015) Cyber Security of Smart Grid Communications: Risk Analysis and Experimental Testing. In: Khaitan S., McCalley J., Liu C. (eds) Cyber Physical Systems Approach to Smart Electric Power Grid. Power Systems. Springer, Berlin, Heidelberg. https://doi.org/10.1007/978-3-662-45928-7 7 .

[10] D. Mah, P. Hills, V.O.K. Li, R. Balme (2014), Smart Grid Applications and Developments, Green Energy and Technology, SpringerVerlag London. https://doi.org/10.1007/978-1-4471-6281-0.

[11] G.M. Shrestha, J. Jasperneite (2012), Performance Evaluation of Cellular Communication Systems for M2M Communication in Smart Grid Applications. In: Kwiecień A., Gaj P., Stera P. (eds) Computer Networks. CN 2012. Communications in Computer and Information Science, vol 291. Springer, Berlin, Heidelberg. https://doi.org/10.1007/978-3-642-31217-5_37.

[12] H. Daki, A.E. Hannani, A. Aqqal, A. Haidine, A. Dahbi (2017), Big Data management in smart grid: concepts, requirements and implementation, Journal of Big Data, vol. 4, no. 13, pp. 1-19. https://doi.org/10.1186/s40537-017-0070-y.

[13] D.S. Markovic, I. Branovic, R. Popovic (2015), Smart Grid and nanotechnologies: a solution for clean and sustainable energy, Energy and Emission Control Technologies, vol. 2015, no. pp. 1-13. https://doi.org/10.2147/EECT.S48124.

[14] Communications Requirements of Smart Grid Technologies, Department of Energy (2010). Available. [Online]: https://www.energy.gov/sites/prod/files/gcprod/documents/Smart_Grid_Communications_Requirements_Report_10-05-2010.pdf

[15] X. Fan, G. Gong (2013), Security Challenges in Smart-Grid Metering and Control Systems, Technology Innovation Management Review, pp. 42-49. Available. [Online]: https://pdfs.semanticscholar.org/b9c3/9b52322c1a3352fc13af70dafb7a5de301a8.pdf

[16] Q. Xu, P. Ren, H. Song, Q. Du (2017), Security-Aware Waveforms for Enhancing Wireless Communications Privacy in Cyber-Physical Systems via Multipath Receptions, IEEE Internet of Things Journal, vol. 4, no. 6, pp. 1924-1933. https://doi.org/10.1109/JIOT.2017.2684221.

[17] Z. ElMrabet, N. Kaabouch, H. ElGhazi, H. ElGhazi (2018), Cybersecurity in smart grid: Survey and challenges, Computers \& Electrical Engineering, vol. 67, pp. 469-482. https://doi.org/10.1016/j.compeleceng.2018.01.015.

[18] Y. Kabalci (2016), a survey on smart metering and smart grid communication, Renewable and Sustainable Energy Reviews, vol. 57, pp. 302-318. https://doi.org/10.1016/j.rser.2015.12.114.

[19] Naamane, N.K. Msirdi (2015), Towards a Smart Grid Communication, Energy Procedia, vol. 83, pp. 428-433. https://doi.org/10.1016/j.egypro.2015.12.162.

[20] Mahmood, N. Javaid, S. Razzaq (2015), A review of wireless communications for smart grid, Renewable and Sustainable Energy Reviews, vol. $41, \quad$ pp. 248-260. https://doi.org/10.1016/j.rser.2014.08.036. 
[21] R. Leszczyna (2018), Cybersecurity and privacy in standards for .smart grids - A comprehensive survey, Computer Standards \& Interfaces, vol. $\quad 56, \quad$ pp. https://doi.org/10.1016/j.csi.2017.09.005.

[22] Y. Wang, T.T. Gamage, C.H. Hauser (2016), Security Implications of Transport Layer Protocols in Power Grid Synchrophasor Data Communication, IEEE Transactions on Smart Grid, vol. 7, no. 2, pp. 807-816. https://doi.org/10.1109/TSG.2015.2499766.

[23] T. Sukumara, J. Starck, J. Vellore, E. Kumar, G. Harish (2018) Cyber security - Securing the protection and control relay communication in substation, 71st Annual Conference for Protective Relay Engineers (CPRE), College Station, TX, pp. 1-7. https://doi.org/10.1109/CPRE.2018.8349788.

[24] G. Artale, et al. (2018), a new low cost power line communication solution for smart grid monitoring and management, IEEE Instrumentation \& Measurement Magazine, vol. 21, no. 2, pp. 29-33. https://doi.org/10.1109/MIM.2018.8327976.

[25] Z. Cai, M. Yu, M. Steurer, H. Li, Y. Dong (2016), A network model for the real-time communications of a smart grid prototype, Journal of Network and Computer Applications, vol. 59, pp. 264-273. https://doi.org/10.1016/j.jnca.2015.05.024

[26] N. Shaukat, S.M. Ali, C.A. Mehmood, B. Khan, M. Jawad, U. Farid, Z. Ullah, S.M. Anwar, M. Majid (2018), A survey on consumers empowerment, communication technologies, and renewable generation penetration within Smart Grid, Renewable and Sustainable Energy Reviews, vol. 81, no. 1, pp. 1453-1475. https://doi.org/10.1016/j.rser.2017.05.208.

[27] R.M. Onoshakpor, K.C. Okafor (2017), Cyber security in smart grid convolution networks (SGCNs), IEEE 3rd International Conference on Electro-Technology for National Development (NIGERCON) Owerri, pp. 392-399. $\quad$ https://doi.org/10.1109/NIGERCON.2017.8281911.

[28] H. Elshaafi, M. Vinyals, I. Grimaldi (2018), S. Davy, Secure Automated Home Energy Management in Multi-Agent Smart Grid Architecture, Technology and Economics of Smart Grids and Sustainable Energy, vol. 3, no. 4, pp. 1-13. https://doi.org/10.1007/s40866-018-0042-0.

[29] M. Abrar, M.A. Tahir, R. Masroor, H.M.U. Hamid (2018), Real time smart grid load management by integrated and secured communication, International Conference on Innovative Trends in Computer Engineering (ITCE), Aswan, pp. 253-257. https://doi.org/10.1109/ITCE.2018.8316634. 